\title{
A Comparative Study on different AI Techniques towards Performance Evaluation in RRM(Radar Resource Management)
}

\author{
Madhusudhan H.S, \\ Assistant Professor, \\ Department of Information Science \& Engineering, \\ VVIET, Mysore, India.
}

\author{
Khalid Nazim S.A, \\ Associate Professor and Head, \\ Department of Information Science \& Engineering, \\ VVIET, Mysore, India.
}

\begin{abstract}
The multifunction radar (MFR) has to make a decision as to which functions are to be performed first or which must be degraded or even not done at all when there are not enough resources to be allocated. The process of making these decisions and determining their allocation as a function of time is known as Radar Resource Management (RRM). The RRM has two basic issues: task prioritization and task scheduling. The task prioritization is an important factor in the task scheduler. The other factor is the required scheduling time, which is decided by the environment, the target scenario and the performance requirements of radar functions. The required scheduling time could be improved by using advanced algorithm $[1,6]$.
\end{abstract}

Keywords- Radar; RRM (Radar Resource Management); Artificial Intelligence (AI); Neural Network (NN); Fuzzy Logic (FL).

\section{INTRODUCTION}

The term RADAR is an acronym for RAdio Detection And Ranging. RADAR's are used in detection and location of objects like spacecraft, aircraft, ships, vehicles, people and natural environment. It uses radio waves to detect objects or targets otherwise invisible because of distance from observer, darkness or naturally occurring barriers such as fog or cloud cover. In addition to detection, radar can determine the object's distance or range from the radar station, its position and its speed and direction of movement [1].

Multifunction radar is based on phased arrays and it is able to execute multiple functions integrated all together. Multifunction radar (MFR) performs many functions previously performed by individual, dedicated radars, such as search, tracking and weapon guidance, etc. The primary interest when looking at the operational efficiency of this type of radar system is to schedule the radar jobs effectively. A detailed functional simulation model, which generates a multifunctional radar environment, has been developed to aid the evaluation of the various scheduling algorithm [6].

Multifunction radar is the main sensor for modern weapon control systems. The radar consists of an electronic beam steering phased antennas and performs surveillance and tracking of multiple targets and simultaneous tracking and guiding of multiple missiles.

The advantage of a phased array radar system over mechanically scanned radar is the provision to put the beam at anytime by electronically switching the beam. This enables adaptive track updates, and instantaneous scheduling of urgent tasks such as confirmation of detection.

The multifunction radar (MFR) has to make a decision as to which functions are to be performed first or which must be degraded or even not done at all when there are not enough resources to be allocated. The process of making these decisions and determining their allocation as a function of time is known as Radar Resource Management (RRM). All the functions or the tasks are coordinated by a central component RRM in the radar system. This RRM component is critical to the success of a MFR since it maximizes the radar resource usage in order to achieve optimal performance $[6,8,9]$.

\section{LITERATURE SURVEY}

\section{A. Introduction to AI techniques:}

In real systems, the use of a fuzzy logic method and neural network method may represent a useful support for radar resource management decisions. It is also very important that the information in respect of radar resource management is presented to the radar operator at all times $[8,9]$.

Fuzzy Logic is a logical system, which is an extension of multivalued logic. However, in a wider sense fuzzy logic (FL) is almost synonymous with the theory of fuzzy sets, a theory which relates to classes of objects with unsharp boundaries in which membership is a matter of degree [2].

Neural networks are composed of simple elements operating in parallel. These elements are inspired by biological nervous systems. As in nature, the connections between elements largely determine the network function. Neural network can be trained to perform a particular function by adjusting the values of the connections (weights) between elements [3, 4].

\section{B. The Radar Resource Management Problem}

There are two major radar resources: time and energy. The challenge of the RRM arises when the radar resources are not enough to assist all the tasks in all the functions. Lower priority tasks must encounter degraded performance due to less available resources, or the radar may not execute some tasks at all. Each task in the radar requires a certain amount of time, energy and computational resource. The time is characterized 
by the tactical requirements, the energy is limited by the transmitter energy, and the RRM limits the computational resource. All of those limitations have impacts on the performance of the radar resource management $[1,6,8,9]$.

An additional challenge is that since the RRM deals with many radar subsystems, evaluation of the RRM algorithms must be done under a more complex and detailed radar model. A general MFR resource management system model is shown in Fig. 1. It performs the following steps:

- Get a radar mission profile or function setup;

- Generate radar tasks;

-Assign priorities to tasks by using a prioritization algorithm;

- Manage available resources by a scheduling algorithm so that the system can meet the requirements of all radar functions;

- The radar scheduler considers radar beams, dwell time, carrier frequency, and energy level, etc.

As can be seen from the above steps, the RRM problem has two basic issues: task prioritization and task scheduling. Some RRM algorithms handle the two issues separately and others handle them simultaneously. The task prioritization is an important factor in the task scheduler. The other factor is the required scheduling time, which is decided by the environment, the target scenario and the performance requirements of radar functions. The required scheduling time could be improved by using advanced algorithms.

A RRM algorithm can be non-adaptive or adaptive. In a non-adaptive scheduling algorithm, the task priorities are predefined and the radar scheduler includes some heuristic rules. Therefore, the resource perf

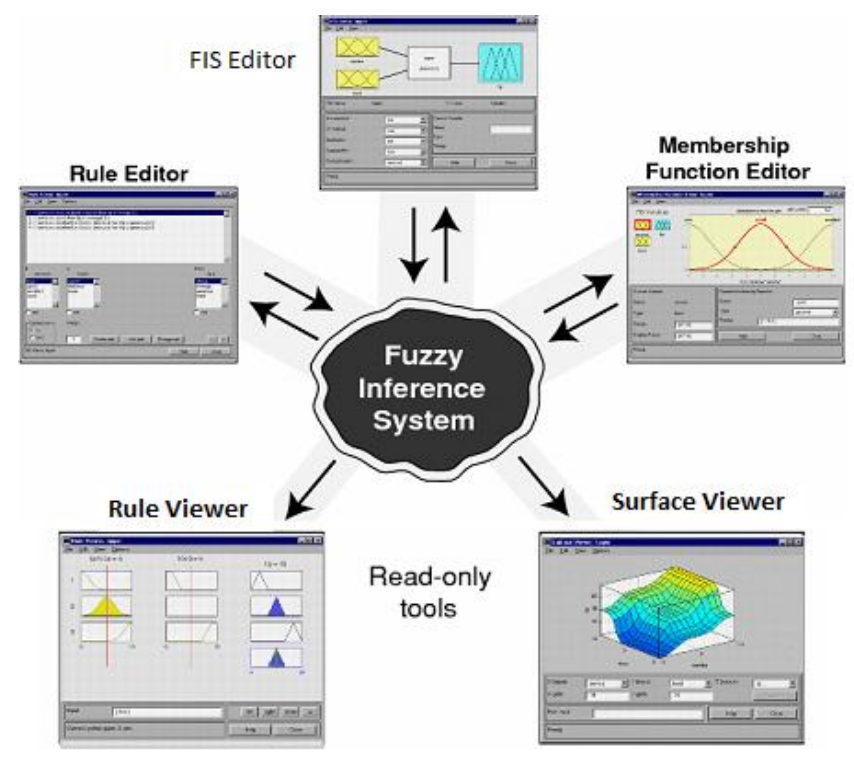

Figure 2. Fuzzy Inference System. ormance is not optimized $[6,9]$.

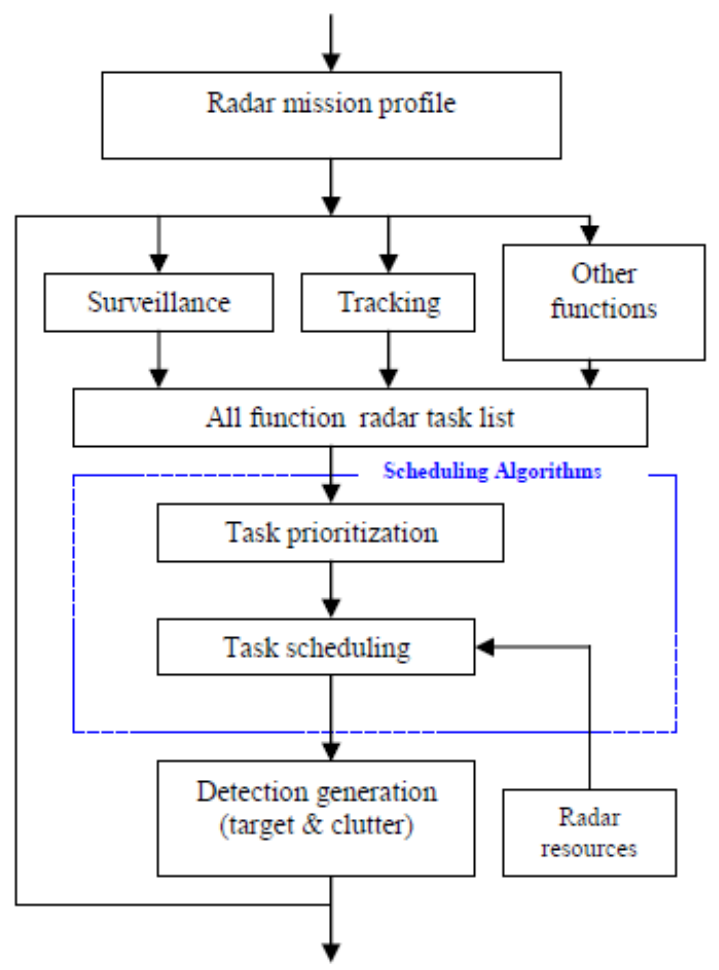

Figure 1. MFR Resource Management Model.

\section{Solution to RRM problem:}

Adaptive scheduling algorithms are much more complex, and should theoretically yield better performance. Advanced MFRs always use adaptive scheduling algorithms. One of the algorithm is Artificial Intelligence (AI) algorithm.

There are 2 algorithms under AI, which are used in task prioritization:

\section{Fuzzy Logic Approach}

2. Neural Network.

1. Fuzzy Logic approach: Fuzzy logic has two different meanings. In a narrow sense, fuzzy logic is a logical system, which is an extension of multivalued logic. However, in a wider sense Fuzzy Logic (FL) is almost synonymous with the theory of fuzzy sets, a theory which relates to classes of objects with unsharp boundaries in which membership is a matter of degree. Fig. 2 shows the FIS (Fuzzy Inference System) that helps in formulating the mapping from the mapping from a given input to an output using FL [2].

2. Neural Network elements are as shown in Fig. 3. Neural networks are adjusted or trained such that a particular input leads to a specific target output $[3,4]$. 


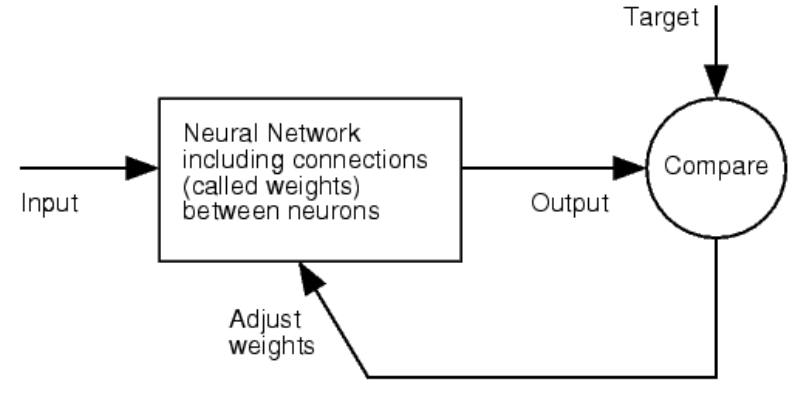

Figure 3. Neural Network

\section{METHODOLOGY}

\section{A. Architecture}

The system architecture is as shown in Fig. 4 that focuses on tracking, surveillance and task scheduling.

The various functions of the system architecture are as discussed in the following section.

a. Surveillance function: Based on the information and on the desired surveillance performance, the surveillance function calculates the number of radar beams necessary to survey that volume. A list of task requests is generated, taking into account the desired surveillance performance of the radar system. The surveillance manager is fed by the task list, maintained an inactive queue of tasks (not yet scheduled), and provides the scheduler with a smaller queue of requests that are close to their due time of execution.

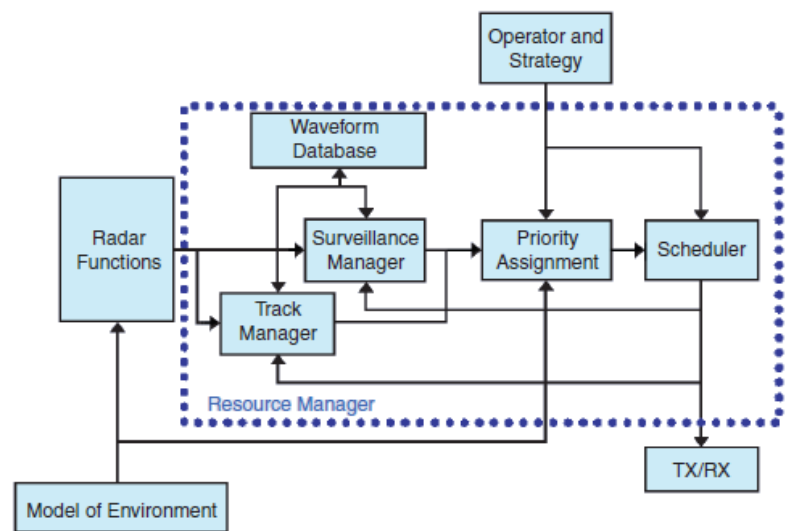

Figure 4. System architecture

b. Track function: calculates the update times of the targets under track and feeds the track manager with a queue of tasks to be scheduled. The track manager also maintains a list of inactive track tasks that are to be sent to the scheduler when close to their due time of execution.

c. Waveform Database: Both surveillance and track manager select from the waveform database the parameters to be used in the transmission of the radar pulses associated with each radar job.

d. Priority Assignment: Decisions relating to how resources are to be allocated according to the relative importance of the tasks and how to assess this relative importance are made by priority assignment block. Here 2 methods are discussed for prioritizing tasks. 1. Prioritization using fuzzy logic. 2. Prioritization using Neural Networks.

e. Scheduler: It is fed by queues of track, plot confirmation, surveillance task requests and creates a set of measurement tasks to be carried out by the radar based on task priorities and time constraints. A feedback loop between the output of the scheduler and the radar functions enables the next update times related to those tasks to be calculated.

f. Operator and Strategy: This module operates as a human-machine interface, allowing intervention to enable corrections in the behavior of the system [8].

\section{B. Prioritization using Fuzzy Logic and Neural Network:}

The attribution of priority to regions and targets of interest is central to the eventual performance of the radar system and to subsequent mission success. There are a variety of methods that may be employed, from simple fixed allocations based on operational experience to more elaborate schemes that attempt to balance competing components that constitute the overall determination of priority. The priority for tracking targets may be evaluated using the decision tree presented in Fig. 5. This could be carried out according to information provided by a tracking algorithm, by other sensors, or by other operational modes of the multifunction radar $[6,9]$.

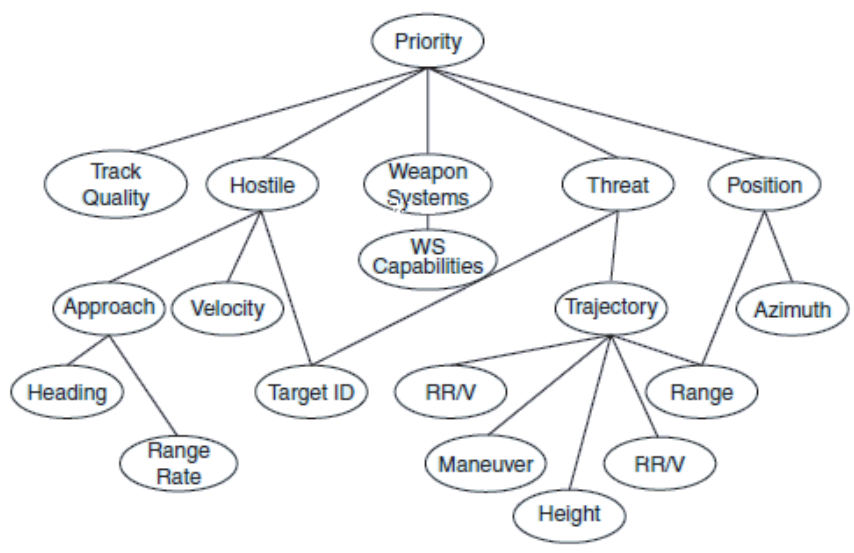

Figure 5. Decision tree for target priority assignment.

Some examples of the fuzzy values are presented in Table I. Five different variables provide information used to set the priority level. These are threat, hostility, quality of tracking and relative position of the target, and weapon system capabilities of the platform. After evaluation of these variables according to a set of fuzzy rules (i.e. FIS is constructed at each level of the tree) the importance (priority) of the target is determined [6, 9, 13].

TABLE I. FUZZY VALUES RELATED TO THE MAIN VARIABLES USED IN THE PRIORITY ASSIGNMENT.

\begin{tabular}{|c|c|}
\hline Fuzzy Variable & Fuzzy values \\
\hline Track Quality & High and Low \\
\hline Hostile & Nonhostile, Unknown And Hostile \\
\hline Weapon Systems & Low And High Capability \\
\hline Threat & Low, Medium ,High And Very High \\
\hline
\end{tabular}




\begin{tabular}{|c|c|}
\hline Position & Close, Medium And Far \\
\hline Priority & Low, Medium, High And Very High \\
\hline
\end{tabular}

A similar methodology is applied to the surveillance function base upon the decision tree presented in Fig. 6. In this case, the priority of surveillance sectors may be assessed through the original priorities attributed to the regions with respect to the expected tactical scenarios and the information gathered during the evolution of the actual environments. This includes aspects such as rate of detection of new targets, number of threatening targets, and rate of detection of new threatening targets. A set of fuzzy rules (a FIS) enables the evaluation of the priority of the different sectors considered for surveillance

$[9$,

13].

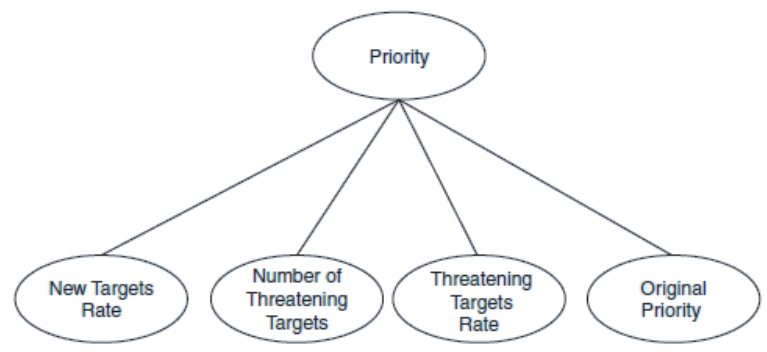

Figure 6. Decision tree for sectors of surveillance priority assessment

In Neural Network approach a Neural Network is constructed at each level of the decision tree (shown in Fig. 5) and the output is fed to the next higher level. This process repeates till priority of the target is calculated. To calculate the priority of sector of surveillance one Neural Network is used [2].

\section{Performance Evalution}

By knowing the identity of the targets, their priorities may vary. This provides valuable information to be accounted for when deciding how to allocate radar resources in overload situations. Three targets are assumed in the analysis, their probabilities of being enemy are different as follows: 1 (enemy), 0.5 (unknown), and 0.1 (friendly), corresponding to the red, blue, and green curves, respectively. The probabilities of targets are kept constant. The evolution of the resulting priorities is shown in Fig. 7 and Fig. 8 for 50 radar scans, which shows that, in general, all priorities increase as the targets move towards the radar platform and the greater the probability of being enemy, the greater the resulting priority.

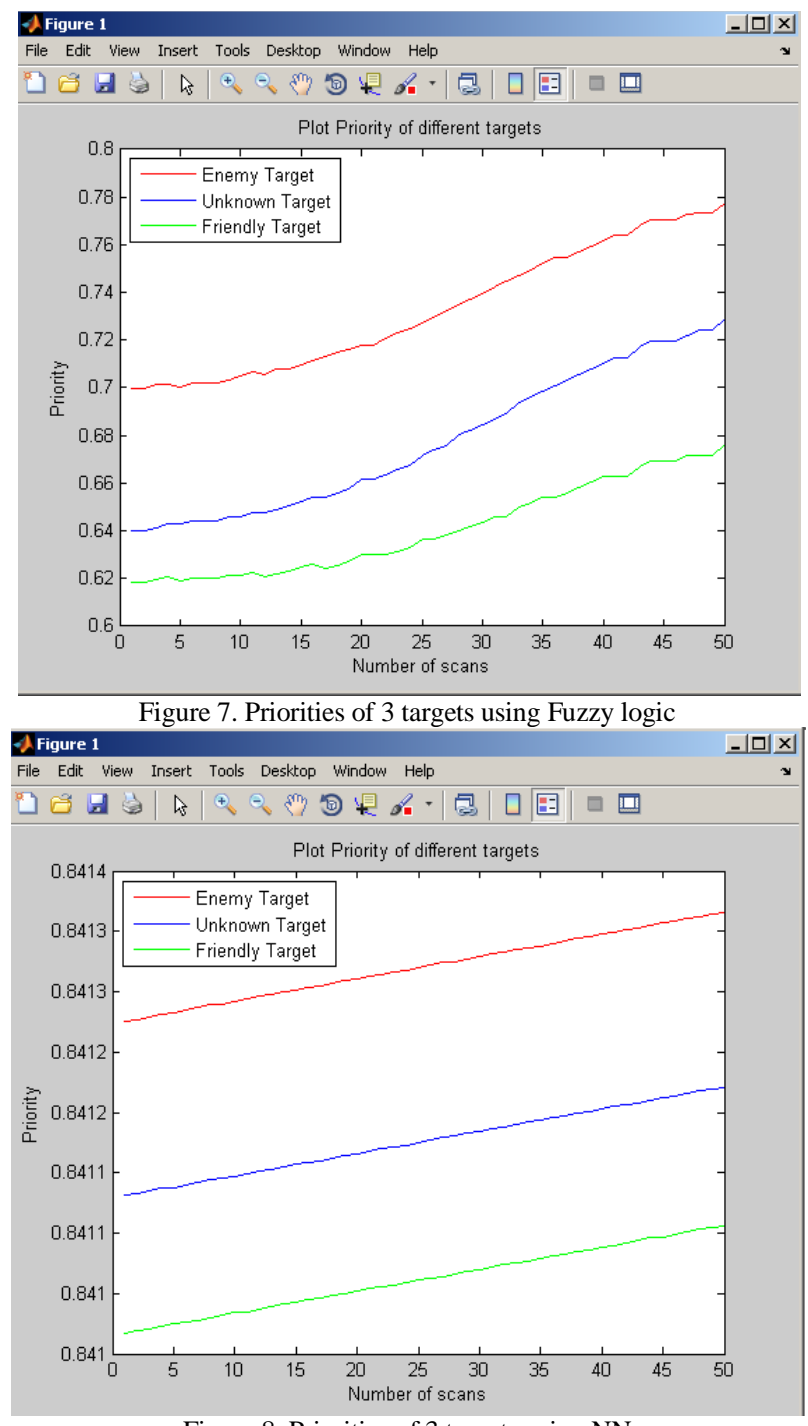

Figure 8. Priorities of 3 targets using NN

Fig.7 and Fig. 8 presents the results of a priorities calculated where targets are assumed to move on a straight line trajectory with constant velocity. From the first scan onwards priorities of targets calculated using neural network is high compare to priorities obtained using fuzzy logic.

At first, there is a tendency to consider that if two systems execute the same set of tasks, the system that assesses these tasks with lower priority should be considered more effective, because fewer resources would be allocated to execute the tasks. The task priority, therefore, is important for preparing the set of measurements to be executed by the radar. Fuzzy logic approach and neural network approach for prioritizing radar tasks in changing environment conditions is introduced. Results suggest that the fuzzy approach is a valid means of evaluating the relative importance of the radar tasks; the resulting priorities have been adapted by the fuzzy logic prioritization method $[6,8,9,13]$.

\section{CONCLUSION}

Prioritization is a key component to determine overall performance. Performance of two scheduling methods is 
compared. Fuzzy logic suggests that fewer resources can be allocated to execute tasks, which is more important for radar to manage resource. Fuzzy approach has operational advantages particularly under stressing conditions, which is important in design of RRM.

In future other AI techniques, such as an entropy algorithm and Expert System Approach can be used to evaluate performance of RRM based on neural networks and fuzzy logic techniques.

\section{REFERENCES}

[1] www.radartutorial.eu

[2] http://www.mathworks.com/products/fuzzylogic/

[3] http://www.learnartificialneuralnetworks.com/

[4] http://www.mathworks.com/products/neuralnet/

[5] Komorniczak, W., Kuczerski, T., Pietrasinski, J.F, "The Priority Assignment for Detected Targets in Multi-function Radar". In 13th International Conference on Microwaves, Radar and Wireless Communications, Wroclaw, Poland, Pp 244-247. Vol.1.2000.

[6] M.T. Vine, "Fuzzy logic in radar resource management," in Multifunction Radar and Sonar Sensor Management Techniques IEEE colloquium Dig., 2001.
[7] Komorniczak, W., Kuczerski, T. and Pietrasinski, J, The priority assignment for detected targets in multifunction radar, Journal of Telecommunications and Information Technology, 30-32, 2001.

[8] S.L.C. Miranda, C.J. Baker, K. Woodbridge, and H.D. Griffiths, "Phased array radar resource management: A comparison of scheduling algorithms," in Proc. 2004 IEEE Radar Conf., Philadelphia, USA, 2004.

[9] S.L.C. Miranda, C.J. Baker, K. Woodbridge, and H.D. Griffiths, "Phased array radar resource management: A fuzzy logic approach for prioritization of radar tasks and sectors of surveillance," in Proc. Radar 2004 Conf., Toulouse, France, Oct. 2004.

[10] G. Zeng, and W. D. Hu, "The simulation on adaptive scheduling for multifunction phased array radars", Journal of System Simulation, Beijing, 16(9), pp. 2026-2029, 2004.

[11] K. Tei-Wei, C. Yung-Sheng, Chin-Fu Kuo, and C. Chang, "Real-time dwell scheduling of component-oriented phased array radars," IEEE Transaction, Jan. 2005.

[12] Miranda, S.L.C., Baker, K., Woodbridge, K. and Griffiths, H.D, Simulation methods for prioritizing tasks and sectors of surveillance in phased array radar, Journal of Simulation, 5 (1-2), 18-25, 2005.

[13] Miranda, S.L.C., Baker, K., Woodbridge, K. and Griffiths, H.D, Knowledge-based resource management for multifunction radar, IEEE Signal Processing Magazine, 66 (1), 66- 76, 2006.

[14] Miranda, S.L.C., Baker, K., Woodbridge, K. and Griffiths, H.D, Fuzzy logic approach for prioritisation of radar tasks and sectors of surveillance in multifunction radar, IET Proceedings - Radar, Sonar and Navigation, 1 (2), 131-141, 2007 